\title{
Smart City Wien als Motor für eine zukunftsfähige Entwicklung Wiens
}

\author{
T. Madreiter, B. Vogl \\ Online publiziert am 26. September 2012 \\ (c) Springer-Verlag 2012
}

Eine Vielzahl von Gründen wie die Klimaveränderung durch fossile Energieträger, die unsichere Zukunft der Versorgungssicherheit und vieles mehr bestärkt die Politik weltweit, Entwicklungen im Klimaschutz- und Energiebereich mit dem Ziel der Steigerung der Energieeffizienz und der Erhöhung des Anteils erneuerbarer Energien voranzutreiben. Bis 2050 strebt die Europäische Union eine Reduktion der Treibhausgase um zumindest $80 \%$ an. Dabei ist vor allem auf die Versorgung städtischer Strukturen mit neuen intelligenten Systemen zu achten, da Städte weiter wachsen werden und ihre Bedeutung somit zunimmt. Bereits heute leben über $50 \%$ der Weltbevölkerung in Städten, und Städte sind für $75 \%$ der weltweiten $\mathrm{CO}_{2}$-Emissionen verantwortlich.

Wien setzt seit langem auf die Verknüpfung von Lebensqualität, Technologie und Umweltschutz. Wien ist daher eine der führende Umweltmusterstädte weltweit bzw. auch in Smart City Rankings ganz vorne zu finden. Wien setzt auf die Weiterentwicklung dieser Führungsrolle und stellt bei seinen Smart City-Aktivitäten die Bereiche Energie, Verkehr und Stadtentwicklung in den Mittelpunkt.

Wien verfolgt im Energie- und Klimabereich ehrgeizige Ziele. In Wien koordiniert die "Magistratsabteilung 20 - Energieplanung" die Umsetzung des "Städtischen Energieeffizienz-Programms (SEP)" und des "Renewable Action Plans Vienna (RAP_Vie)", die Meilensteine der Wiener Energiepolitik darstellen.

Das SEP rückt Energieeffizienz und Energiesparen in den Vordergrund und gibt Leitlinien für die verbraucherseitige Energiepolitik bis zum Jahr 2015 vor. Eine Fortsetzung des SEP ab 2015, mit längerfristigen energiepolitischen Perspektiven, ist geplant. Im Lichte der angestrebten Reduktion von Treibhausgasen um mindestens $80 \%$ bis 2050 müssen unsere Energieversorgungssysteme und -technologien deutlich effizienter werden.

Der Renewable Action Plan Vienna (RAP_Vie) wird derzeit gerade erarbeitet und soll Ende des Jahres im Gemeinderat beschlossen werden. Es werden im RAP_Vie Möglichkeiten und Maßnahmen für die Weiterentwicklung erneuerbarer Energien in Wien festgelegt werden. Bedeutende Potenziale für die Entwicklung Erneuerbarer, die noch nicht erschlossen sind, sind v. a. in den Bereichen Photovoltaik, Solarwärme, Geothermie, Grundwasserwärme und Erdwärme vorhanden. Ganz wesentlich wird in dem Zusammenhang die Frage des Umbaus der Netzinfrastruktur inklusive der Möglichkeit, auch große Mengen von Überschussstrom aus Wind-, Solar- und anderen erneuerbaren Energiekraftwerken zu speichern, sein.

Aus den beiden Strategien ergeben sich, nochmal zusammenfasst, die energiepolitischen Grundlagen für die Smart City Wien mit folgenden wesentlichen Säulen:

- Effizienter Umgang mit Energie

- Erneuerbare in Wien weiter ausbauen

- An das Angebot erneuerbarer Energien angepasste Verbrauchsstrukturen durch intelligente Steuerung und Technologieauswahl

- Speichertechnologien entwickeln und ins System integrieren

Die „Magistratsabteilung 18 - Stadtentwicklung und Stadtplanung" überarbeitet zurzeit den Stadtentwicklungsplan (STEP). In diesem Prozess der Erarbeitung des STEP'14 werden die für die Stadtentwicklung relevanten Aspekte des Smart City (SC)-Ansatzes kohärent eingebracht. Dies bedeutet, den SC-Ansatz als Arbeitsprinzip in die Stadtentwicklungsplanung festzulegen, die davon angesprochenen Themenfelder zu definieren und konkrete Zielsetzungen sowie Maßnahmen abzuleiten. Damit soll gewährleistet werden, dass die mit dem STEP'14 zu verbindenden Smart City-Umsetzungsaktivitäten der Stadt Wien vorbereitet werden. Der neue Stadtentwicklungsplan soll 2014 vom Gemeinderat beschlossen werden.

Im Verkehrsbereich setzt man in der Verkehrs- und Stadtplanung auf den weiteren Ausbau der öffentlichen Verkehrsmittel in Kombination mit Fahrrad und zu Fuß gehen. Ein Strategie für E-Mobility ist gerade in Entwicklung.
Kurzfassung eines Vortrags der 50. Fachtagung der Österreichischen Gesellschaft für Energietechnik (OGE) im OVE, gemeinsam mit den Energietechnischen Gesellschaften in Electrosuisse und im VDE, die am 10. und 11. Oktober 2012 in Wien stattfindet.

Madreiter, Thomas, Dipl.-Ing., Magistrat der Stadt Wien, MA 18, Stadtentwicklung und Stadtplanung, Rathausstraße 14-16, 1082 Wien, Österreich; Vogl, Bernd, Mag. Magistrat der Stadt Wien, MA 20, Energieplanung, Amerlingstraße 11, 1060 Wien, Österreich (E-Mail: thomas.madreiter@wien.gv.at) 